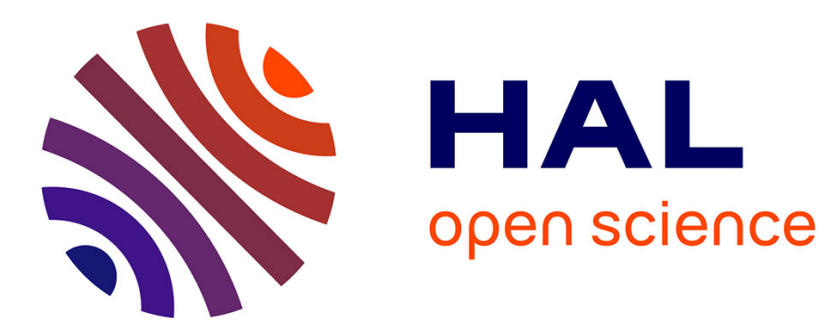

\title{
Magnetic phase diagram of small superconducting two-loop networks
}

\author{
J. Riess
}

\section{To cite this version:}

J. Riess. Magnetic phase diagram of small superconducting two-loop networks. Journal de Physique Lettres, 1982, 43 (8), pp.277-283. 10.1051/jphyslet:01982004308027700 . jpa-00232046

\section{HAL Id: jpa-00232046 https://hal.science/jpa-00232046}

Submitted on 1 Jan 1982

HAL is a multi-disciplinary open access archive for the deposit and dissemination of scientific research documents, whether they are published or not. The documents may come from teaching and research institutions in France or abroad, or from public or private research centers.
L'archive ouverte pluridisciplinaire HAL, est destinée au dépôt et à la diffusion de documents scientifiques de niveau recherche, publiés ou non, émanant des établissements d'enseignement et de recherche français ou étrangers, des laboratoires publics ou privés. 


\title{
Magnetic phase diagram of small superconducting two-loop networks
}

\author{
J. Riess \\ Centre de Recherches sur les très Basses Températures, C.N.R.S., B.P. $166 \mathrm{X}$, \\ 38042 Grenoble Cedex, France
}

(Reşu le 28 janvier 1982, accepté le 22 février 1982)

\begin{abstract}
Résumé. - Le comportement critique de micro-circuits supraconducteurs - systèmes simples à deux boucles en forme de pont de Wheatstone - est calculé en présence d'un champ magnétique. Sur la base du formalisme de de Gennes et Alexander on montre, comment les diagrammes de phase sont obtenus. Les lignes de séparation des phases ont une forme ondulatoire pour des systèmes à boucles suffisamment symétriques et s'apparentent au cas d'une boucle simple. Une interprétation physique en termes de vitesses est donnée, et des expériences nouvelles sont proposées.
\end{abstract}

\begin{abstract}
The critical magnetic behaviour of simple superconducting micro-networks is illustrated by discussing several two-loop systems in the form of Wheatstone bridges. Using the formalism of de Gennes and Alexander it is shown how phase boundaries are obtained. These show undulating behaviour for sufficiently symmetric loop systems, analogous to that of a simple loop. A physical interpretation in terms of velocities is given and new experiments are proposed.
\end{abstract}

1. Introduction. - There is a growing interest in the theory of magnetic properties of superconducting micro-networks [1-3], see also [4]. This theoretical activity has been stimulated by experimental results on percolation networks of granular superconductors (see references quoted in [1]). Networks made of thin superconducting wires (or films) show interesting forms of phase diagrams when they are exposed to an external magnetic field. In this article we illustrate these phenomena by discussing the example of two loops connected by a common boundary (Fig. 1). Our analysis may be helpful for the discussion of similar geometrical systems.

As in references [1-4] we assume the thickness $d$ of the network branches to be much smaller than the penetration depth $\lambda(T)$. This means that the magnetic field created by the induced supercurrents in the loops can be neglected, and further, that in a first approximation the system can be treated as if $d$ were infinitely small. Hence, on each intersection-free branch of the system the superconducting order parameter $\Delta(s)$ depends only on the curvilinear parameter $s$, denoting the distance from a fixed point on the branch.

We will calculate the critical magnetic fields of the loop system in figure 1 (restricting ourselves to second order phase transitions). These fields can be determined from the solutions of the non-linear Ginzburg-Landau equation

$$
\left(i(\partial / \partial s)-(2 e / \hbar c) A_{\|}(s)\right)^{2} \Delta(s)-\left(1 / \xi^{2}(T)\right) \Delta(s)+\left(2 m / \hbar^{2}\right) \beta|\Delta|^{2} \Delta(s)=0
$$




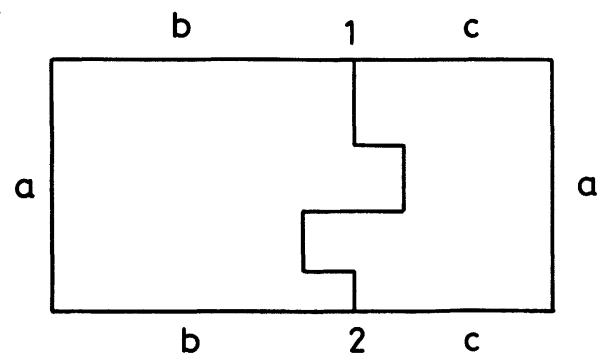

Fig. 1. - The two-loop system discussed is shown. In the figure the inner branch is situated in the plane defined by the outer branches, but it may also lie in a perpendicular plane. This does not change the equations in the text.

where $A_{\|}(s)$ is the tangential component of the vector potential $\mathbf{A}$ associated with the external magnetic field $H=\operatorname{curl} A$, and $\xi(T)$ is the bulk superconducting coherence length at the temperature $T$. (We have defined $2 e>0$.) The critical magnetic fields are obtained as those fields, for which the solution $\Delta(s)$ of equation (1) vanishes continuously as a function of the field.

Alternatively the critical fields (for a second order phase transition) are obtained as those fields $H$, for which the linear Ginzburg-Landau equation (Eq. (1) without the cubic term) has a solution. The latter method will be used in the present article (as in Refs. [2, 3]).

In reference [3] the linear Ginzburg-Landau equation for an arbitrary network has been brought into a convenient mathematical form. We briefly review this derivation by considering an intersection-free branch of an arbitrary network with the endpoints of the branch denoted by $j$ and $k$. On such a branch the general solution $\Delta(s)$ of the linear Ginzburg-Landau equation is

$$
\Delta(s)=\exp \left(-i \int_{j}^{s}(2 e / \hbar c) A_{\|} \mathrm{d} s\right)\left[p \mathrm{e}^{i s / \xi}+q \mathrm{e}^{-i s / \xi}\right] .
$$

It is convenient to express the undetermined coefficients $p$ and $q$ in terms of $\Delta_{j}$ and $\Delta_{k}$, the (unknown) values of $\Delta(s)$ at the endpoints $j$ and $k$ of the branch. One obtains

$$
\Delta(s)=\exp \left(-i \int_{j}^{s}(2 e / \hbar c) A_{\|} \mathrm{d} s\right)\left[\Delta_{j} \sin \left(\theta_{j k}-s / \xi\right)+\Delta_{k} \mathrm{e}^{i \gamma_{j k}} \sin (s / \xi)\right] / \sin \theta_{j k} .
$$

Here $s$ is the curvilinear coordinate on the branch $(j k)$ measured from point $j$,

$$
\theta_{j k}=l_{j k} / \xi(T),
$$

where $l_{j k}$ is the length of the branch, and

$$
\gamma_{j k}=\int_{j}^{k}(2 e / \hbar c) A_{\|} \mathrm{d} s .
$$

For each branch the unknown values $\Delta_{j}, \Delta_{k}$ are determined by the boundary conditions ( Kirchhoff-conditions ») at the endpoints of the branch (Eq. (3) of Ref. [2], Eq. (4) of Ref. [3]). This leads to the following set of coupled equations [3] :

$$
\sum_{k}\left\{\left(-\Delta_{j}\right) \operatorname{cotg} \theta_{j k}+\Delta_{k} \mathrm{e}^{i i_{j k} / \sin \theta_{j k}}\right\}=0 .
$$


(The summation over $k$ means the sum over all branches $(j k)$ branching off at $j$, where $k$ are nodes nearest to $j$.) Equation (6) is equivalent to the linear Ginzburg-Landau equation on the total network.

Equation (6) represents a homogeneous linear system for the $\Delta_{j}$. There exists at least one $\Delta_{j}$, which cannot be determined from (6), and to which all other $\Delta_{k}$-values are proportional. This $\Delta_{j}$-value itself goes to zero at the second order phase transition as a consequence of the nonlinear Ginzburg-Landau equation. (For a simple loop the exact solution of the non-linear Ginzburg-Landau equation is known for each flux value through the loop (cf. e.g. Ref. [5], p. 187, or the discussion in [4]), and the equivalence of the phase transitions obtained from the linear and the non-linear Ginzburg-Landau equations can easily be verified.)

2. The two-loop system (Wheatstone bridge). - If we apply equation (6) to the network shown in figure 1 , the following linear system of equations results

where

$$
\begin{aligned}
-\alpha \Delta_{1}+\beta \Delta_{2} & =0 \\
\beta^{*} \Delta_{1}-\alpha \Delta_{2} & =0,
\end{aligned}
$$

$$
\begin{aligned}
& \alpha=\operatorname{cotg} \theta_{l}+\operatorname{cotg} \theta_{i}+\operatorname{cotg} \theta_{r}, \\
& \beta=\mathrm{e}^{i \gamma_{l}} / \sin \theta_{l}+1 / \sin \theta_{i}+\mathrm{e}^{i \gamma_{r}} / \sin \theta_{r} .
\end{aligned}
$$

Here $\Delta_{1}, \Delta_{2}$ are the (unknown) values of the order parameter at the intersections 1 and $2, \theta_{l}$, $\theta_{i}, \theta_{r}$ are the lengths of the left hand, inner and right hand branches divided by $\xi(T)$

$$
\text { (e.g., } \left.\quad \theta_{l}=(a+2 b) / \xi(T)\right) \text {, }
$$

and $\gamma_{l}, \gamma_{i}, \gamma_{r}$ are defined according to (5) (where $j=1, k=2$ ). We take a gauge such that $\gamma_{i}=0$. This is achieved e.g. with a Landau-gauge $A=\left(0, H_{x}, 0\right)$, where $H$ is the external magnetic field perpendicular to the plane of the network. Hence $\gamma_{l}\left(-\gamma_{r}\right)$ is the magnetic flux through the left (right) hand part of the loop system, and $\phi=\gamma_{l}-\gamma_{r}$ is the total flux. Equations (7), (8), (9) are formally invariant under any deformation of the loop system for which $\gamma_{i}$ remains zero. (E.g., the outer branches may be segments of circles.)

We first consider the symmetric geometry $\left(b=c\right.$, i.e. $\left.\theta_{l}=\theta_{r}=\theta / 2, \gamma_{l}=-\gamma_{r}=\phi / 2\right)$. In this case $\beta$ is real and equation (7) has the following two types of solutions :

a) symmetric solution $\Delta_{1}=\Delta_{2}$, which is obtained from the compatibility equation

$$
\alpha=\beta
$$

b) antisymmetric solution $\Delta_{1}=-\Delta_{2}$, obtained from the compatibility equation

$$
\alpha=-\beta \text {. }
$$

The compatibility equations are the condition for the existence of solutions $\Delta_{1}, \Delta_{2}$ of the linear system (7). Numerical solutions of (10) and (11) are shown in figure $2 a$ (solid lines correspond to symmetric, dashed lines to antisymmetric solutions). These lines represent points in the $(\phi, \theta)$-plane, where the individual solutions $\Delta(s)$ of the non-linear Ginzburg-Landau equation (1) vanish.

For points $(\phi, \theta)$ between the $\phi$-axis and the nearest line of solutions (full or broken line, whatever comes first) the system is in the normal state, since here no solution of the non-linear Ginzburg-Landau equation (1) exists. Thus the left hand boundary of the line pattern represents the phase boundary $\phi(\theta)$ for the second order phase transition between the normal and the superconducting states. (We remember that $\theta$ is the total circumference of the loop system divided by $\xi(T)$.) In figure $2 a$ the phase boundary is a sequence of line sections which alternately 


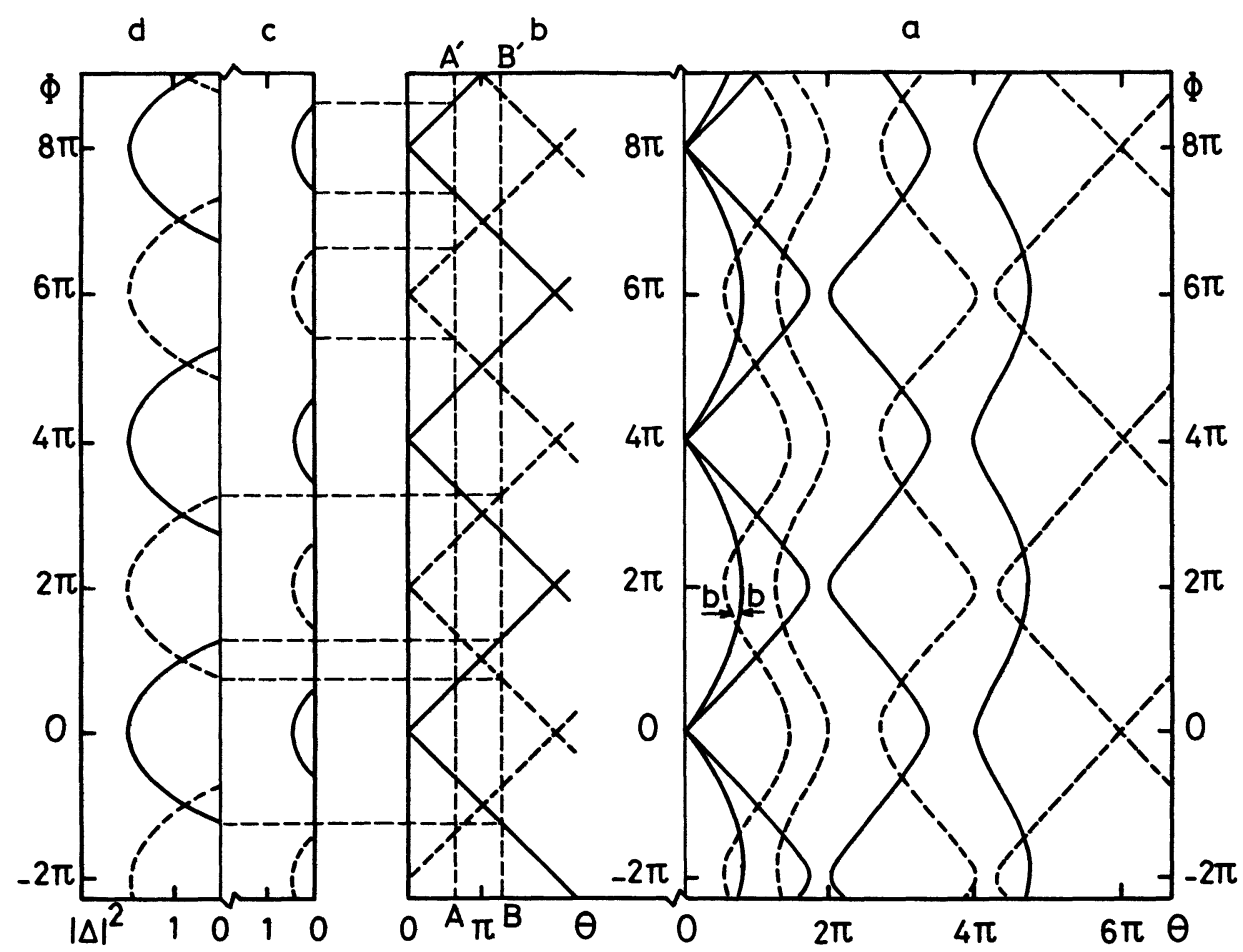

Fig. 2. - - a) Critical lines obtained from equations (10) (solid lines) and (11) (broken lines), corresponding respectively to symmetric and antisymmetric solutions of equation (7) for the system shown in figure 1, with the geometry $a=b=c$, and a length of the inner branch equal to $a$. The two lines marked by $b$ are the corresponding first solutions (for lowest $\theta$-values) for the geometry $a=b=c$, and a length of the inner branch equal to $6 a . \phi$ is the total flux through the loop system (in units of $\hbar c / 2 e$ ). $\theta$ is the total outer circumference divided by $\xi(T)$. Note the symmetry with respect to $\phi=0, \pm 2 \pi, \pm 4 \pi, \ldots b$ ) Critical lines for a simple loop, representing symmetric (full lines) and antisymmetric (dashed lines) solutions of equation (6) for a simple loop. c) Square of the order parameter $|\Delta|^{2}$ along the line $\mathrm{AA}^{\prime}$ of figure $2 b$ (in units of $\hbar^{2}\left(2 \beta m L^{2}\right)$, with $L=$ circumference of the loop). $d$ ) The same as figure $2 c$, but along BB' of figure $2 b$.

represent the symmetric (full line) and antisymmetric (broken line) solutions of the linear system (7).

The form of the phase boundary varies with the geometry of figure 1 . The two lines denoted by the letter $b$ are the first two solutions for the geometry $\theta_{i}=2 \theta_{l}$. All other lines in figure $2 a$ represent solutions of the case $\theta_{i}=\theta_{l} / 3$. We see that the total superconducting area is larger in the first case.

In reference [3] two limits of equations (10) and (11) are discussed : for $\theta_{i} \rightarrow 0$ essentially the equation of two independent simple loops is obtained, whereas for $\theta_{i} \rightarrow \pi$ the equation for a loop with no internal branch is approached. (We remark, that the second limit does not describe a physical critical line, since it is situated beyond the phase boundary, as can be seen from figure $2 a$, case $a=b=c$, where $\theta_{i} \rightarrow \pi$ implies $\theta \rightarrow 6 \pi$.)

It is illustrating to compare figure $2 a$ with the pattern of critical lines (Fig. $2 b$ ) of a simple loop (omitting the inner branch in figure 1). Here the structure of the lines is simpler and, moreover, the corresponding solutions of the non-linear Ginzburg-Landau equation are known (illustrated in figures $2 c, d$ ). In particular we see that along the line $\mathbf{B B}^{\prime}$ of figure $2 b$ adjacent solutions of the non-linear Ginzburg-Landau equation overlap, whereas along the line $\mathrm{AA}^{\prime}$ this is not the case. 
Thus if $\phi$ is increased on the line $\mathbf{B B}^{\prime}$ the system is always superconducting, but the order-parameter and the free energy vary periodically with $\phi$, with a period $\Delta \phi=2 \pi(\phi$ in units of $\hbar c / 2 e)$. Along the line $\mathrm{AA}^{\prime}$ however the superconducting and the normal state alternate.

The situation of the two-loop system (Fig. $2 a$ ) is somewhat similar to that of a simple loop, at least for sufficiently small $\theta$-values (i.e., for the left hand part of the line pattern in figure $2 a$ ). It is useful to imagine the behaviour of the order parameter in that area. In the vicinity of $\theta=0$, $\phi=2 \pi m, m$ even integer ( $\phi$ in units of $\hbar c / 2 e$ ), the solution of equation (10) mathematically approaches the corresponding symmetric solution of a simple loop. This is illustrated by the similar line form in figures $2 a$ and $2 b$. Therefore the order parameters of the two systems must roughly resemble each other in that area. If we exclude the behaviour on the inner branch of the two-loop system, this rough similarity of the order parameters should also be true in the vicinity of $\phi=2 \pi m, m$ odd integer, and $\theta=\theta_{1}$, where $\theta_{1}$ is the smallest value of the antisymmetric solutions in figure $2 a$ (for a definite geometry).

The phase boundary $\phi(T)$ can be measured in an experiment of Little-Parks type [6]. For a two-loop system adjacent maxima of the transition temperature are not identical as a consequence of the difference between symmetric and antisymmetric solutions (Fig. $2 a$ ), in contrast to a simple loop (Fig. $2 b$ ).

Figure $2 a$ suggests two further types of experiments. First let us fix the geometry and the temperature such that $0<\theta<\theta_{1}$. (In figure $2 a$ the $\phi$-values belonging to $\theta_{1}$ are equal to $2 \pi \times$ odd integer.) If one increases the magnetic field, the system alternates periodically between the superconducting and the normal state, with a period $\Delta \phi=4 \pi$ (corresponding, in ordinary flux units, to twice the quantum of flux, i.e., to $2(\hbar c / 2 e)$ ), in contrast to the same experiment made with a simple loop, where the period is $\Delta \phi=2 \pi$, since $\theta_{1}=0$.

Next the temperature $T$ is lowered such that $\theta_{1}<\theta(T)<\theta_{2}$, where $\theta_{2}$ is defined as the smallest value of intersection between symmetric and antisymmetric solutions. (For the case $\theta_{i}=2 \theta_{l}$, e.g., $\theta_{2}$ is defined by the intersection of the lines marked by the letter $\mathrm{b}$ in figure $2 a$.) When $\phi$ is increased, the system alternates between the superconducting and the normal state similar to a simple loop. However, for a two-loop system adjacent superconducting areas are not identical, i.e., one has a superposition of two periodic behaviours, each of period $\Delta \phi=4 \pi$, one associated with the symmetric, the other with the antisymmetric solutions. For $\theta>\theta_{2}$ the system is superconducting for any flux i.e., there is no critical magnetic field (in our approximation).

Let us replace in figure $2 a$ the flux variable $\phi$ by $\gamma=\phi / 2$, corresponding to the flux through only one of the two symmetrical loops. Now the distance between identical points on the phase boundary is equal to $\Delta \gamma=2 \pi$. This suggests the following experimental situation : we perform a Little-Parks experiment for a simple loop (here adjacent minima of the transition temperature are a quantum of flux apart). After that we "glue " to it a second, identical loop to obtain the symmetric case of figure 1 and repeat the experiment. Then additional minima appear halfway between those of a simple loop (i.e., the distance between adjacent minima is now half a quantum of flux).

We next consider the asymmetric case of figure $1(b \neq c)$. Here the compatibility equations are

$$
\alpha= \pm|\beta|
$$

leading to the two types of solution

$$
\Delta_{2}= \pm \Delta_{1} \mathrm{e}^{-i \varphi_{\beta}}
$$

where $\varphi_{\beta}$ is the phase of the complex number $\beta$ defined by equation (9) (in the symmetric geometry $\beta$ is real). In figure 3 numerical solutions of (12) for the case $a=c=b / 2$ are shown. Here the phase boundary is entirely given by the first symmetric solution (full line), corresponding to the plus sign of equation (12). The first antisymmetric solution (dashed line) does not form a 


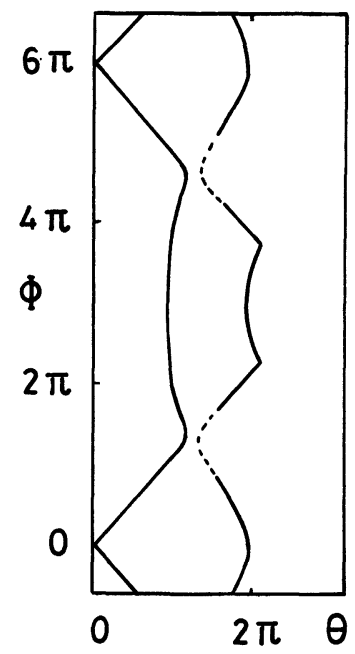

Fig. 3. - Critical lines of the first symmetric (solid line) and the first antisymmetric (broken line) solutions of equation (7) for the asymmetric case of figure $1(c=a, b=2 a$, the length of the inner branch is equal to $a)$. The solutions are periodic with a period $\Delta \phi=6 \pi$.

connected line, suggesting jumps of the corresponding solutions of the non-linear GinzburgLandau equation.

If the thickness $d$ of the wires is taken into account, the phase boundary is shifted to lower temperatures (higher $\theta$ ). The envelope of the oscillating phase boundary curves in figures $2 a$ and $2 b$ are given by (cf. e.g. Eq. (10) of Ref. [4])

$$
\phi \approx(\sqrt{3} L \theta) /(2 \pi d)
$$

( $\phi$ in units of $(\hbar c / 2 e), L$ is the total circumference of the loop system). This is a straight line beginning at $\phi=0, \theta=0$ and ending at $\phi(\theta(T=0)), \theta(T=0)$. Equation (14) has been derived for a simple loop, but it should also be valid for the two-loop system due to the similarity (for $d=0$ ) of the two order parameters in the vicinity of critical points on the envelope ( $\phi$-axis). A thorough discussion of the phase boundaries of a hollow cylinder of finite wall thickness, including superheating and supercooling effects is given in [7].

3. Currents and velocities. - The usual definition of the current (e.g., Eqs. (6)-(12) of Ref. [5]) applied to equation (3) gives

$$
j_{j k}=(2 e \hbar) /(m \xi)\left|\Delta_{j}\right|\left|\Delta_{k}\right| \sin \left(\varphi_{j}-\varphi_{k}-\gamma_{j k}\right) / \sin \theta_{j k}
$$

$\left(\varphi_{j}, \varphi_{k}\right.$ are the phases of the order parameters $\Delta_{j}=\left|\Delta_{j}\right| \mathrm{e}^{i \varphi_{j}}$, and $\left.\Delta_{k}=\left|\Delta_{k}\right| \mathrm{e}^{i \varphi_{k}}\right)$. Equation (15) has the form of a Josephson current through a weak link. (We remark that the general form (3) of $\Delta(s)$ implies relations between the order parameters and its first spatial derivatives at both endpoints $j, k$, which are of the same general form as the corresponding relations for the order parameters on both sides of a weak link, leading directly to the expression for the Josephson current. Compare e.g. [5], chapt. 7-3.)

At a second order phase transition the order parameter and hence the currents $j_{j k}$ vanish. We therefore define a velocity $v_{j k}$ by dividing the current by $2 e|\Delta|^{2}$. At the phase boundary $v_{j k}$ is called critical velocity.

If a magnetic field is applied to a superconducting micro-network, velocities are induced in 
the loops. With increasing field the system either reaches the critical velocity and becomes normal, or it jumps to another solution of the non-linear Ginzburg-Landau equation. This new solution has a lower free energy (in a certain field interval) due to a non-zero fluxoid along one or more of the loops, giving rise to a proper velocity, which partly or totally cancels the induced velocity. For higher fields another solution with different fluxoid quantum numbers becomes energetically more favourable, a. s. o. (see e.g. Figs. $2 b, c, d$ ).

Whenever the phase boundary has a locally highest transition temperature, the free energy has a local minimum corresponding to a local minimum of the critical velocity. This is illustrated by our two-loop systems : in the symmetric case $(b=c)$ we have $\varphi_{1}-\varphi_{2}=0$ or $\pi$. Hence on the inner branch, where $\gamma_{12}=0$, the velocities $v_{12}$ vanish. On the outer branches they oscillate with the flux $\gamma_{12}=\frac{1}{2} \phi$, becoming zero for $\phi=2 \pi \times$ integer, corresponding to local maxima of the transition temperature. For the asymmetric geometry we have $\varphi_{1}-\varphi_{2}=\varphi_{\beta}$ or $\varphi_{\beta}+\pi$. Since $\varphi_{\beta}$ is generally different from zero, the velocities through both the inner and outer branches are non-zero in general. This explains in figure 3 the disappearance of the local temperature maxima at $\phi=(2 \pi, 4 \pi) \times$ integer present in the symmetric case of figure $2 a$. For a totally asymmetric loop system the phase boundary is aperiodic. This means that (due to velocities in the loops which never completely vanish) the bulk transition temperature is never attained, except for zero field.

Acknowledgments. - We thank R. Maynard for helpful discussions.

\section{References}

[1] De Gennes, P. G., C.R. Hebd. Séan. Acad. Sci. Paris 292, série II (1981) 9.

[2] DE Gennes, P. G., C.R. Hebd. Séan. Acad. Sci. Paris 292, série II (1981) 279.

[3] Alexander, S., preprint (1981).

[4] Riess, J., J. Low Temp. Phys. 47, to be published.

[5] DE Gennes, P. G., Superconductivity of Metals and Alloys (Benjamin, N.Y.) 1966.

[6] Little, W. A. and Parks, R. D., Phys. Rev. Lett. 9 (1962) 9 ; Phys. Rev. 133 A (1964) 97.

[7] FInk, H. J. and GrüNFeld, V., Phys. Rev. B22 (1980) 2289. 